\title{
Exchange Bias and Coercivity in Vicinity of Magnetic Compensation Point in GdFeCo Amorphous Film
}

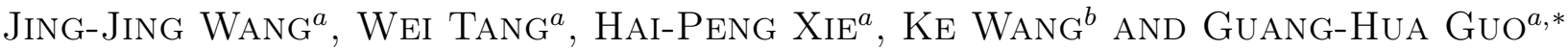 \\ ${ }^{a}$ School of Physics and Electronics, Central South University, Changsha 410083, China \\ ${ }^{b}$ School of Mechanical and Electronic Engineering, East China University of Technology, \\ Nanchang 330013, China
}

(Received September 9, 2019; revised version November 27, 2019; in final form January 2, 2020)

Magnetic properties of amorphous GdFeCo film are studied in the temperature range from 5 to $300 \mathrm{~K}$. The temperature dependence of the magnetization shows that the GdFeCo film has different compensation temperature for cooling and heating processes. Perpendicular magnetic anisotropy is observed in the GdFeCo film and persists in the whole measuring temperature range. Coercivity increases rapidly as the temperature approaches the compensation point and displays M-type variation. The hysteresis loop shows a small exchange bias at room temperature. The exchange bias is observed to increase significantly when temperature is close to the compensation point and shows multiple peak variation. Two magnetic phases are suggested to be coexisting in the sample. Bases on this assumption, the peculiar magnetic properties of the amorphous GdFeCo film can be well explained.

DOI: 10.12693/APhysPolA.137.368

PACS/topics: rare earth, transition metal amorphous film, exchange bias, coercivity, magnetic compensation temperature

\section{Introduction}

Exchange bias, referring to the shift of hysteresis loop along the magnetic field axis, was first found by Meiklejohn and Bean in $\mathrm{Co} / \mathrm{CoO}$ core-shell particle system [1]. This phenomenon has attracted a great deal of attention recently due to its technological applications in spintronics devices [2-4]. Although the mechanism of exchange bias is not yet fully understood, it is widely accepted that the exchange bias is due to the symmetry breaking of magnetization reversal process. The symmetry breaking is usually caused by the interfacial exchange interaction between a ferromagnet (FM) and an antiferromagnet (AFM) as in FM/AFM bilayers (multilayers) [5-10] or FM/AFM composite granular films [11-13]. The exchange bias effect is also observed in some intermetallic compounds and alloys, such as $\mathrm{Ni}-\mathrm{Mn}, \mathrm{Fe}-\mathrm{Mn}, \mathrm{Co}-\mathrm{Mn}$ binary alloys $[14,15]$. The coexistence of FM and AFM phases or spin glass phase is regarded as the origin of the exchange anisotropy. In soft/hard ferromagnetic bilayers, the interfacial exchange interaction at the interface of the hard and soft layers also gives rise to the bias of the minor hysteresis loop of the soft layer $[16,17]$. The symmetry of magnetization reversal process can be broken by the exchange interaction between the local magnetic moments and the spin polarized conduction electrons in light/heavy rare earth ferrimagnetic alloys [18, 19]. Near the magnetic compensation point, the oppositely directed local magnetic moments cancel each other out, and the

*corresponding author; e-mail: guogh@mail.csu.edu.cn conduction electron polarization has a significance contribution to the magnetization [20]. The exchange coupling between the local moments and the conduction electrons makes the hysteresis loop asymmetric [18]. Recently, a giant exchange bias of more than $3 \mathrm{~T}$ was reported in a family of the Heusler alloys with a compensated ferrimagnetic state [21]. The large exchange anisotropy originates from the exchange interaction between the compensated host and anti-site disorder induced ferromagnetic clusters.

Heavy rare earth (HRE)-transition metal (TM) amorphous films such as GdFeCo and $\mathrm{TbFeCo}$ films are well known bulk perpendicular magnetic anisotropic (PMA) materials [22-28]. Comparing to PMA films induced by interface effect, the PMA in HRE-TM amorphous films remain even in films with thickness over tens of nanometers, therefore, the PMA is thermally more stable for HRE-TM amorphous films. HRE-TM amorphous films also have strong magneto-optical effect $[22,29]$. These characteristics make the HRE-TM amorphous films ideal materials for information storage and processing. Moreover, all-optical induced ultrafast magnetization switching on picosecond time scales was realized in some HRE-TM films [30-32].

In this paper, we prepare GdFeCo amorphous films with PMA by using magnetron sputtering method. The magnetic properties are measured in the temperature range from 5 to $300 \mathrm{~K}$. Results show that the exchange bias and coercivity exhibit peculiar behavior in the vicinity of magnetic compensation point. The exchange bias is enhanced greatly. A two phase model is used to explain the complex temperature behavior of the GdFeCo amorphous films. 


\section{Experimental detail}

GdFeCo films were sputtered at room temperature in a chamber with a base pressure down to $6 \times 10^{-6} \mathrm{~Pa}$. Before deposition, $10 \mathrm{~nm}$ Ta was pre-sputtered on $\mathrm{Si}(100)$ substrate as buffer layer. GdFeCo films were rf sputtered based on a composite target method, as we described before [33]. The working pressure of Ar gas was fixed at $0.6 \mathrm{~Pa}$ and the sputtering power was $80 \mathrm{~W}$ during the sputtering of GdFeCo alloy films. The thickness of GdFeCo films was about $20 \mathrm{~nm}$. No protective capping layer is used after deposition of GdFeCo.

The structure of the GdFeCo films was characterized by a X-ray diffractometer (XRD, Rigaku Smartlab) with $\mathrm{Cu} K_{\alpha}$ radiation. Composition of the samples was determined by X-ray photoelectron spectroscopy (XPS). In order to measure the atomic composition in different depths of the film, the surface of sample was sputtered by argon ion for different times. The power for ion sputtering is $3 \mathrm{keV}$. Magnetic properties were measured by commercial Physical Property Measurement System (Quantum Design).

\section{Results and discussion}

Figure 1 presents the full XPS spectra of GdFeCo film and the spectra for $\mathrm{Gd} 3 d_{5 / 2}$, Fe $2 p_{3 / 2}$, Co $2 p$, and $\mathrm{O} 1 s$ before and after argon ion sputtering. The spectrum before ion sputtering shows that the surface of the film is severely oxidized. The O $1 s$ peak located at ca. $532.35 \mathrm{eV}$ is introduced by surface contaminant due to the ex situ. The peak at $c a .530 .13 \mathrm{eV}$ can be assigned to $\mathrm{MO}_{x}(\mathrm{M}=\mathrm{Gd}, \mathrm{Fe}$, and $\mathrm{Co})$. It is difficult to distinguish the $\mathrm{GdO}_{x}, \mathrm{FeO}_{x}$, and $\mathrm{CoO}_{x}$ because of their almost same positions of $\mathrm{O} 1 s$ core level. The existence of $\mathrm{GdO}_{x}$, $\mathrm{FeO}_{x}$, and $\mathrm{CoO}_{x}$ are also confirmed by fitting the spectra for $\mathrm{Gd} 3 d_{5 / 2}, \mathrm{Fe} 2 p_{3 / 2}$, and Co $2 p_{1 / 2}$. After 20 and $40 \mathrm{~min}$ argon ion sputtering, the oxidization is greatly reduced, and the spectra mainly reflect the Gd $3 d_{5 / 2}$, $\mathrm{Fe} 2 p_{3 / 2}$ and Co $2 p_{3 / 2}$ core level peaks. The composition of the film is estimated to be $\mathrm{Gd}_{18} \mathrm{Fe}_{76} \mathrm{Co}_{6}$. The XPS spectra also show the compositional inhomogeneous in thickness. Gd atoms tend to migrate to the surface. This phenomenon was also reported in [34].

In HRE-TM amorphous films, the magnetic moments of the rare-earth ions are antiparallel to the moments of the transition metal. The PMA forms in the films with composition in the vicinity of the compensated ferrimagnetic state [35]. For the GdFeCo samples studied in this work, the FeCo magnetic sublattice is dominated at room temperature. The temperature dependence of the magnetization measured under a field of 500 Oe normal to the film plane is shown in Fig. 2. With decreasing temperature, the Gd magnetic moment increases more rapidly than the FeCo moment, and as a result, a compensation point $T_{\text {comp }}$ at which the moments of Gd and FeCo counteract each other appears on the $M-T$ curve. Below $T_{\text {comp }}$, the net magnetic moment is parallel to the moment of Gd-sublattice. It is worth noting that the
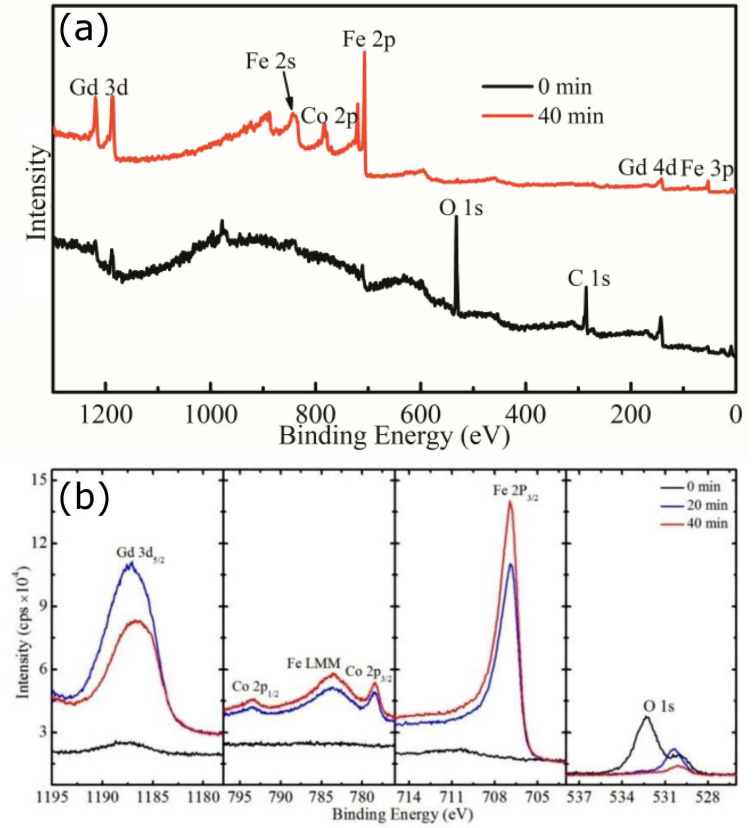

Fig. 1. Full XPS spectra of GdFeCo film (a) and the spectra for $\mathrm{Gd} 3 d_{5 / 2}$, Fe $2 p_{3 / 2}$, Co $2 p$, and $\mathrm{O} 1 s$ before and after argon ion bombardment (b).

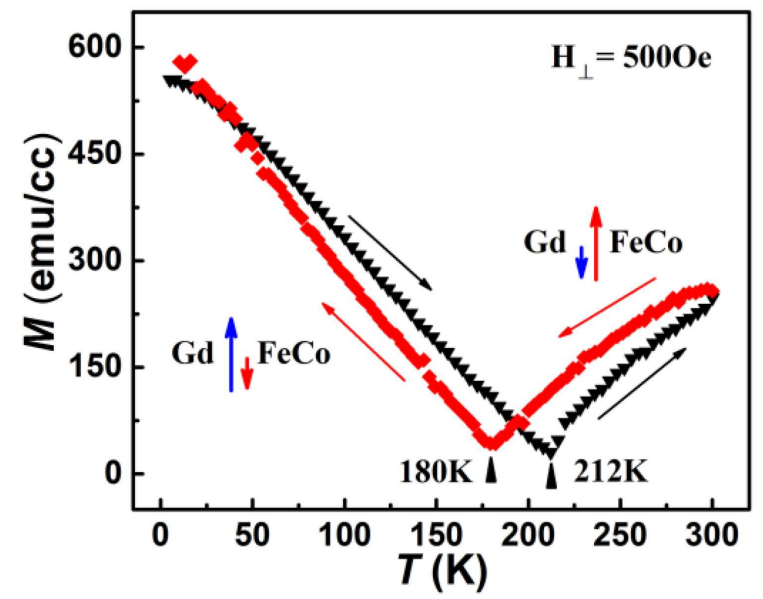

Fig. 2. Temperature dependence of the magnetization of $\mathrm{GdFeCo}$ sample measured at magnetic field of $500 \mathrm{Oe}$ normal to the film plane. The red and blue arrows represent the moments of FeCo- and Gd-sublattices, respectively.

compensation point is different for the cooling and heating processes. For the cooling process $T_{\text {comp }}=180 \mathrm{~K}$, while it is $212 \mathrm{~K}$ for the heating process. Later we will discuss this peculiar phenomenon in more detail.

Figure 3 shows the hysteresis loops measured at different temperatures while the magnetic field is applied perpendicular to the film plane. The maximum field is 2000 Oe and the field scanning step is 2 Oe. The room temperature hysteresis loop is first measured. 

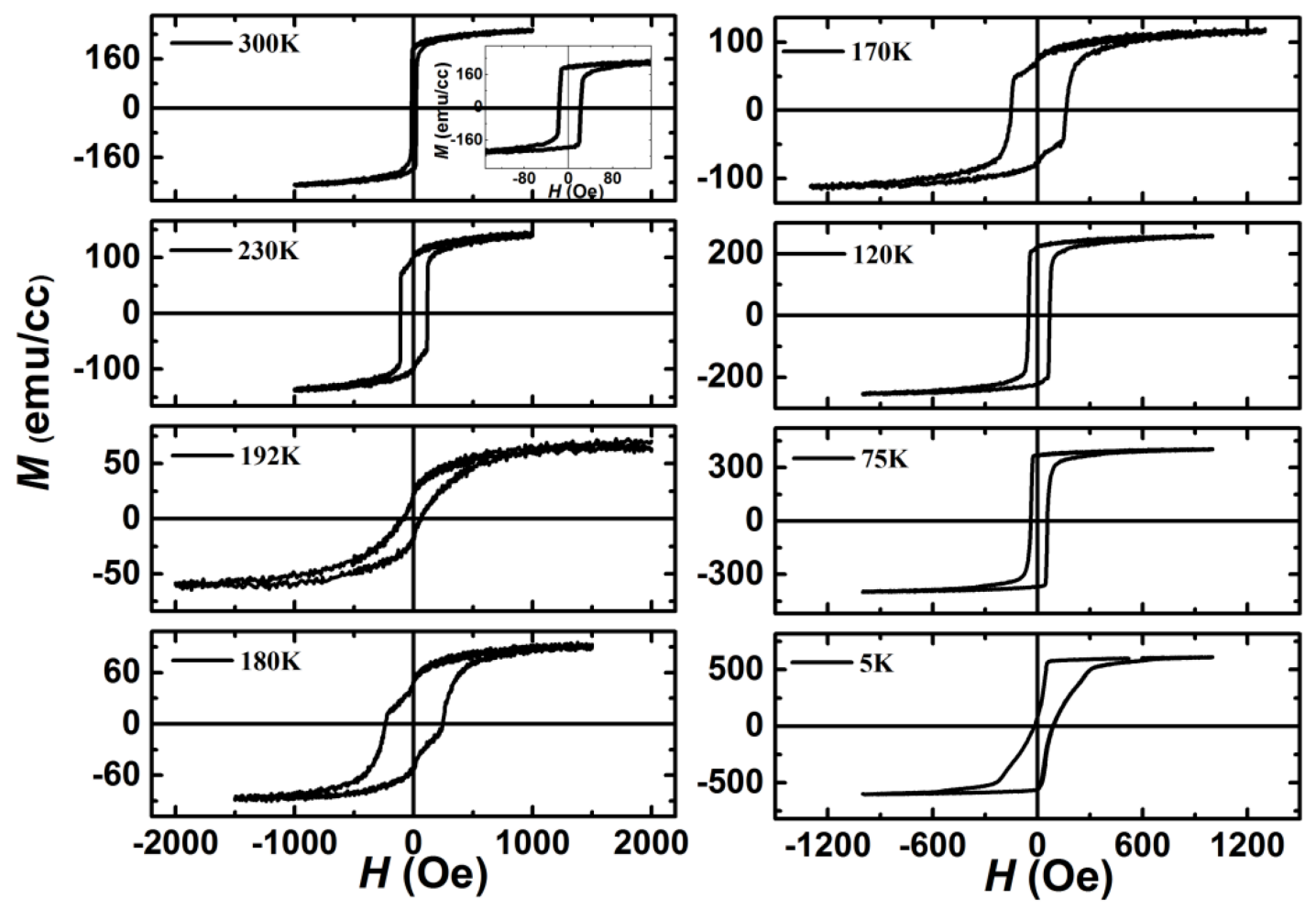

Fig. 3. Magnetization hysteresis loops of GdFeCo amorphous film measured at different temperatures. The magnetic field is applied perpendicular to the film plane.

After that, the sample is cooled down and the loops at different temperatures are measured from $5 \mathrm{~K}$ to room temperature. At room temperature, the hysteresis loop has a good rectangular shape with a coercivity of about 19 Oe and a saturation magnetization of $250 \mathrm{emu} / \mathrm{cm}^{3}$. A very small exchange bias field of 6 Oe is also observed. The rectangular hysteresis loop indicates that the prepared GdFeCo film has PMA. The PMA holds until the lowest temperature as the remanence ratio is close to the unit at most of temperatures. At $5 \mathrm{~K}$, no unit remanence ratio is due to the relatively large positive exchange bias. Actually, one of the remanence equals the saturation magnetization. In the vicinity of the compensation point, the coercivity and the exchange bias field are enhanced greatly.

The temperature dependence of the coercivity is presented in Fig. 4a. The coercivity increases rapidly with decreasing temperature and exhibits a M-type variation in the vicinity of the compensation point as shown in Fig. 4a. Two peaks appear at around $T=183$ and $207 \mathrm{~K}$, respectively. Between them there is a sharp dip at about $194 \mathrm{~K}$. In the very low temperature range $(T<75 \mathrm{~K})$, the coercivity increases slightly. The M-type variation of the coercivity of a ferrimagnet in the vicinity of compensation point was reported in [18, 36]. For ferrimagnet, there are two contributions to the magnetization reversal process when temperature is near the compensation point. One is the switching of the magnetization which is accomplished through the rotation of magnetization or movement of domain wall. Another originates from the antiferromagnetic magnetization process $M=\chi_{a f} H$, i.e., the field-induced change of the values of two sublattice magnetizations or nonlinear structure of two sublattice magnetizations. As this kind of magnetization process exists mainly in antiferromagnet, we denote it as antiferromagnetic magnetization process. Usually, the antiferromagnetic magnetization process is negligible for a ferrimagnet. The coercivity arising from the switching of the magnetization can be approximately determined by $H_{c}=2 K_{u} / \mu_{0} M_{s}-M_{s}$. Here, $K_{u}$ is uniaxial anisotropic constant, $M_{s}$ is saturation magnetization, and $\mu_{0}$ is vacuum permeability. When the temperature approaches the compensation point, $M_{s}$ decreases, which leads to the rapid increase of the coercivity. When temperature is very near the compensation point, the saturation magnetization is very small, and the sample can be regarded as a quasi-antiferromagnet. In this case, the antiferromagnetic magnetization process dominates, and the coercivity drops rapidly and a sharp dip appears as the coercivity due to this contribution is zero [36]. It has to be pointed out that the temperature corresponding to the minimum coercivity is $194 \mathrm{~K}$, neither the cooling compensation point $(180 \mathrm{~K})$ nor the heating compensation point $(212 \mathrm{~K})$, but between them.

The exchange bias also displays complex temperature dependence, as shown in Fig. 4b. At room temperature a small positive exchange bias field is observed. It may come from the exchange interaction between the GdFeCo 

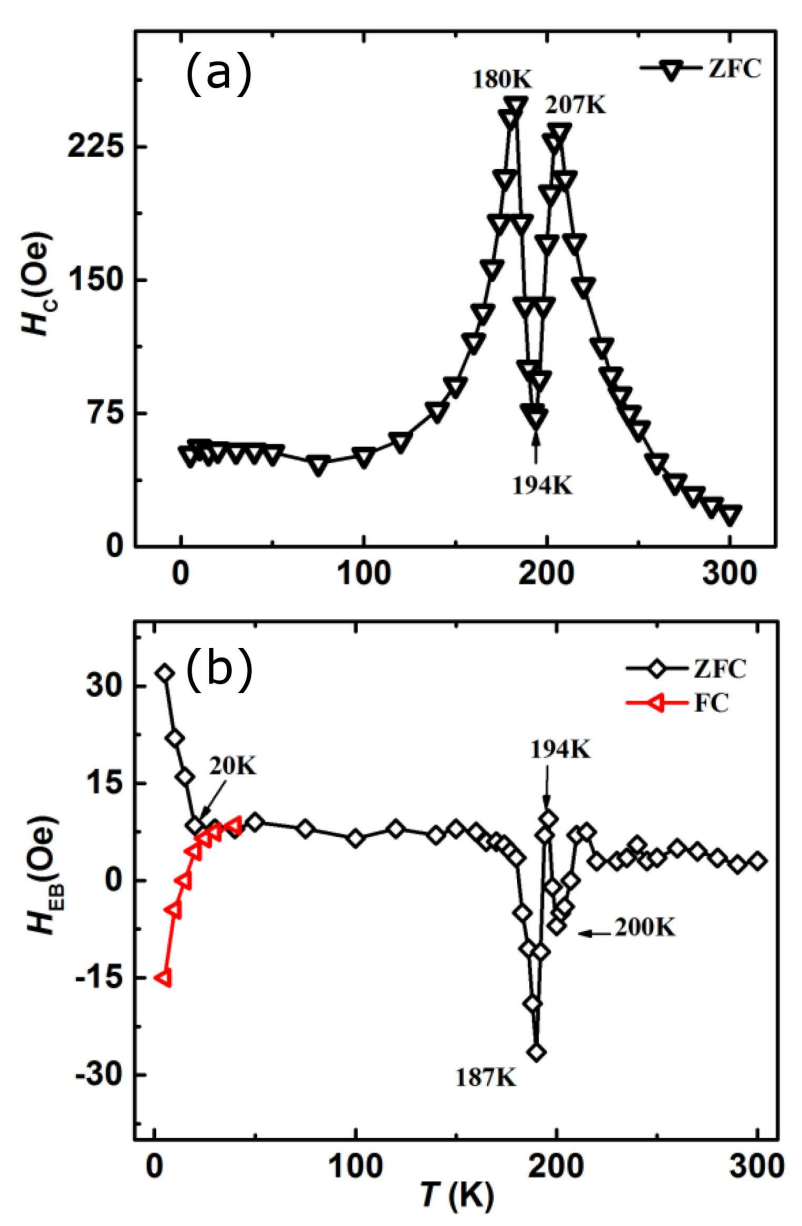

Fig. 4. (a) Temperature dependence of coercivity, and (b) exchange bias of GdFeCo amorphous film.

host and the $\mathrm{CoO}_{x}$ due to the surface oxidation. Our XPS measurements demonstrate the existence of $\mathrm{CoO}_{x}$. The Néel temperature of $\mathrm{CoO}$ in rock salt structure is $293 \mathrm{~K}$, lower than near room temperature. While in the presence of oxygen deficiency, $\mathrm{CoO}_{x}$ may be in the zinc blende or wurtzite crystal structures with Néel temperature higher than room temperature [37]. In the vicinity of the compensation point, the bias field is enhanced greatly and exhibits a multiple peak variation. When temperature is lower than $20 \mathrm{~K}$, the exchange bias increases rapidly again with decreasing temperature. This low temperature exchange bias is related to the cooling process: the field cooling or zero-field cooling. It is positive if the sample is cooled down from room temperature to $5 \mathrm{~K}$ in the absence of magnetic field. When a magnetic field larger than the coercivity is applied during the cooling process, a negative exchange bias field is observed.

The structure and magnetic properties of HRE-TM amorphous films are strongly dependent on the fabrication conditions [38, 39]. The films prepared by RF magnetron sputtering are often compositionally inhomogeneous due to a shadowing effect if separate sources are used for multitarget cosputter deposition [40]. The compositional inhomogeneity in our GdFeCo samples is demonstrated by the XPS spectra (see Fig. 1). The inhomogeneity may give rise to the coexistence of two or more nanoscale magnetic phases in samples [40]. An evidence for the multiple magnetic phases in GdFeCo sample is the two-step magnetization reversal process in some hysteresis loops, as shown in Fig. 3. Here we adopt a twophase model. Based on this model the peculiar variation of the exchange bias in the vicinity of the compensation point can be understood and the different compensation temperature for cooling and heating processes is explained.

Let us assume two magnetic phases, a FeCo-rich phase (labeled as phase I) and a Gd-rich phase (phase II), that coexist in the GdFeCo sample. In phase II, the $\mathrm{Gd}$ moment dominates in the whole temperature range and its saturation magnetization $M_{s}^{\mathrm{II}}=M_{\mathrm{Gd}}^{\mathrm{II}}-M_{\mathrm{FeCo}}^{\mathrm{II}}$ increases with decreasing temperature. Therefore, phase II can be regarded as a ferromagnetic phase [24]. In phase I, the FeCo moment prevails at room temperature and $M_{s}^{\mathrm{I}}=M_{\mathrm{FeCo}}^{\mathrm{I}}-M_{\mathrm{Gd}}^{\mathrm{I}}$. Decreasing temperature Gd moment increases rapidly and exceeds the FeCo moment when temperature is lower than the intrinsic compensation temperature $T_{\text {comp }}^{\mathrm{I}}$ of phase $\mathrm{I}$, therefore $M_{s}^{\mathrm{I}}=M_{\mathrm{Gd}}^{\mathrm{I}}-M_{\mathrm{FeCo}}^{\mathrm{I}}$. The total magnetization is

$$
M_{s}=p^{\mathrm{I}} M_{s}^{\mathrm{I}}+p^{\mathrm{II}} M_{s}^{\mathrm{II}}=p^{\mathrm{I}}\left(M_{\mathrm{FeCo}}^{\mathrm{I}}-M_{\mathrm{Gd}}^{\mathrm{I}}\right)+p^{\mathrm{II}} M_{s}^{\mathrm{II}}
$$

when the sample is cooled from room temperature (cooling process), and

$$
M_{s}=p^{\mathrm{I}} M_{s}^{\mathrm{I}}+p^{\mathrm{II}} M_{s}^{\mathrm{II}}=p^{\mathrm{I}}\left(M_{\mathrm{Gd}}^{\mathrm{I}}-M_{\mathrm{FeCo}}^{\mathrm{I}}\right)+p^{\mathrm{II}} M_{s}^{\mathrm{II}}
$$

for heating the sample from $5 \mathrm{~K}$ (heating process). Here, $M_{\mathrm{FeCo}}^{\mathrm{I}(\mathrm{II})}$ and $M_{\mathrm{Gd}}^{\mathrm{I}(\mathrm{II})}$ represent the magnetizations of FeCo and $\mathrm{Gd}$ in phase I (II), $p^{\mathrm{I}}$ and $p^{\mathrm{II}}$ represent volume concentration of phase I and II, respectively. At the compensation point, the total magnetization of the sample should be zero, i.e.,

$$
\begin{aligned}
& p^{\mathrm{I}}\left(M_{\mathrm{FeCo}}^{\mathrm{I}}-M_{\mathrm{Gd}}^{\mathrm{I}}\right)+p^{\mathrm{II}} M_{s}^{\mathrm{II}}=0 \quad \text { (cooling), } \\
& p^{\mathrm{I}}\left(M_{\mathrm{Gd}}^{\mathrm{I}}-M_{\mathrm{FeCo}}^{\mathrm{I}}\right)+p^{\mathrm{II}} M_{s}^{\mathrm{II}}=0 \quad \text { (heating). }
\end{aligned}
$$

Correspondingly, the $\mathrm{Gd}$ magnetization in phase $\mathrm{I}$ at compensation point can be determined by

$$
\begin{aligned}
& M_{\mathrm{Gd}}^{\mathrm{I}}\left(T_{\text {comp }}^{\text {cooling }}\right)=M_{\mathrm{FeCo}}^{\mathrm{I}}+\left(\frac{p^{\mathrm{II}}}{p^{\mathrm{I}}}\right) M_{s}^{\mathrm{II}} \text { (cooling), } \\
& M_{\mathrm{Gd}}^{\mathrm{I}}\left(T_{\text {comp }}^{\text {heating }}\right)=M_{\mathrm{FeCo}}^{\mathrm{I}}-\left(\frac{p^{\mathrm{II}}}{p^{\mathrm{I}}}\right) M_{s}^{\mathrm{II}} \text { (heating). }
\end{aligned}
$$

Both Eqs. (3) and (4) indicate that $M_{\mathrm{Gd}}^{\mathrm{I}}\left(T_{\text {comp }}^{\text {cooling }}\right)>$ $M_{\mathrm{Gd}}^{\mathrm{I}}\left(T_{\text {comp }}^{\text {heating }}\right)$, meaning the compensation temperature for cooling process is lower than that for heating process, i.e., $T_{\text {comp }}^{\text {cooling }}<T_{\text {comp }}^{\text {heating }}$. Additionally, as $M_{\mathrm{Gd}}^{\mathrm{I}}\left(T_{\mathrm{comp}}^{\mathrm{I}}\right)=M_{\mathrm{FeCo}}^{\mathrm{I}}$, the intrinsic compensation temperature $T_{\text {comp }}^{\mathrm{I}}$ for phase I should be between $T_{\text {comp }}^{\text {cooling }}$ and $T_{\text {comp }}^{\text {heating }}$. That is why the minimum coercivity on $H_{c}-T$ curve is located neither at the cooling compensation point $(180 \mathrm{~K})$ nor at the heating compensation point $(212 \mathrm{~K})$, but between them — namely at $194 \mathrm{~K}$. 
When temperature approaches the intrinsic compensation point $T_{\text {comp }}^{\mathrm{I}}$, phase $\mathrm{I}$ is insensitive to the magnetic field as $M_{s}^{\mathrm{I}}=M_{\mathrm{FeCo}}^{\mathrm{I}}-M_{\mathrm{Gd}}^{\mathrm{I}}$ is very small and equals zero at $T=T_{\text {comp }}^{\mathrm{I}}$. The hysteresis loops mainly come from the contribution of phase II. The exchange coupling between phase I and II at the boundary provides a large exchange anisotropy, which leads to a rapid increase of the exchange bias field. When temperature crosses $T_{\text {comp }}^{\mathrm{I}}$, the direction of magnetization $M_{s}^{\mathrm{I}}$ of phase I is reversed, i.e., the exchange anisotropy reverses. Therefore, the bias field changes its sign. As a result, the exchange bias varies from a negative maximum value to a positive maximum value in the vicinity of the compensation point. While multiple peaks are observed in the vicinity of the compensation point as shown in Fig. 4b. One possible reason is that the migration of the Gd across the thickness may introduce more than two magnetic phases in the sample, and there are two or more intrinsic compensation points which leads to the multiple peaks of the exchange bias field.

The rapid increase of the exchange bias at temperatures below $20 \mathrm{~K}$ originated from interfacial exchange coupling between the GdFeCo host and antiferrmagnetic phases $\mathrm{CoO}_{x}$ and $\mathrm{GdO}_{x}$. But the $\mathrm{GdO}_{x}$ plays the major role as the temperature is in accordance with the GdO Néel temperature (18 K) [41]. The XPS spectra confirm the existence of $\mathrm{GdO}_{x}$ at surface layer. The antiferromagnetic coupling between the Gd moment in $\mathrm{GdO}_{x}$ phase and the FeCo moment in GdFeCo host at the phase boundary gives rise to a large exchange bias. If the sample is cooled down in the absence of magnetic field, the $\mathrm{Gd}$ moment of $\mathrm{GdO}_{x}$ at the interface is antiferromagnetically coupled to the net magnetization of $\mathrm{GdFeCo}$ host, and the exchange bias field is positive. On the contrary, a negative bias field will be obtained if the sample is cooled down in a magnetic field larger than the coercive field as the interface Gd moment in $\mathrm{GdO}_{x}$ is ferromagnetically coupled to the net magnetization of $\mathrm{GdFeCo}$ host.

\section{Conclusions}

We systematically study the magnetic properties of RF sputtered GdFeCo amorphous film in the temperature range from $5 \mathrm{~K}$ to $300 \mathrm{~K}$. The temperature dependence of magnetization shows a compensation point at which the Gd magnetization counteracts the FeCo magnetization. But different compensation temperatures are observed for cooling and heating processes. The hysteresis loops show that the GdFeCo film has PMA in the whole temperature range. The coercivity increases rapidly when temperature approaches the compensation point and exhibits a M-type variation in the vicinity of compensation temperature. At room temperature the hysteresis loop shows a small exchange bias. The exchange bias is enhanced greatly when temperature is near the compensation point and shows a multiple peak variation. When the temperature is lower than $20 \mathrm{~K}$, the exchange bias field increases again. We propose a two magnetic phase model to explain the magnetic properties. The compositional inhomogeneity introduces two magnetic phases, one Gd-rich phase and other one FeCorich phase coexisting in the sample. The Gd-rich phase behaves like a ferromagnetic phase, while the FeCo-rich phase exhibits ferrimagnetic characteristic and shows the compensation phenomenon. Due to the coexistence of the two phases, the compensation temperature at which the net magnetization is zero is different for cooling and heating processes. When temperature is near the compensation temperature, the FeCo-rich phase behaves like an antiferromagnet. The exchange interaction between the FeCo-rich and the Gd-rich phases at boundary gives rise to a larger exchange bias. This kind of exchange bias shows an multiple peak variation when temperature crosses the compensation point. The results provide a new way to manipulate the exchange bias and coercivity, which is significant for magnetic devices.

\section{Acknowledgments}

This work was supported by the National Natural Science Foundation of China (No. 11674400 and 11374373).

\section{References}

[1] W.H. Meiklejohn, C.P. Bean, Phys. Rev. 102, 1413 (1956).

[2] S.S.P. Parkin, K.P. Roche, M.G. Samant, et al., J. Appl. Phys. 85, 5828 (1999).

[3] I. Zutic, J. Fabian, S. Das Sarma, Rev. Mod. Phys. 76, 323 (2004).

[4] S. Parkin, S.H. Yang, Nat. Nanotechnol. 10, 195 (2015).

[5] N. Gökemeijer, T. Ambrose, C. Chien, Phys. Rev. Lett. 79, 4270 (1997).

[6] S. Maat, K. Takano, S.S. Parkin, E.E. Fullerton, Phys. Rev. Lett. 87, 087202 (2001).

[7] Z.Y. Liu, S. Adenwalla, Phys. Rev. Lett. 91, 037207 (2003).

[8] M.R. Fitzsimmons, P. Yashar, C. Leighton, I.K. Schuller, J. Nogues, C.F. Majkrzak, J.A. Dura, Phys. Rev. Lett. 84, 3986 (2000).

[9] J. Camarero, J. Sort, A. Hoffmann, J.M. GarciaMartin, B. Dieny, R. Miranda, J. Nogues, Phys. Rev. Lett. 95, 057204 (2005).

[10] W. Kuch, L.I. Chelaru, F. Offi, J. Wang, M. Kotsugi, J. Kirschner, Nat. Mater. 5, 128 (2006).

[11] J. Nogues, V. Skumryev, J. Sort, S. Stoyanov, D. Givord, Phys. Rev. Lett. 97, 157203 (2006).

[12] L. Del Bianco, D. Fiorani, A. Testa, E. Bonetti, L. Signorini, Phys. Rev. B 70, 052401 (2004).

[13] V. Skumryev, S. Stoyanov, Y. Zhang, G. Hadjipanayis, D. Givord, J. Nogués, Nature 423, 850 (2003).

[14] A.E. Berkowitz, K. Takano, J. Magn. Magn. Mater. 200, 552 (1999). 
[15] S. Giri, M. Patra, S. Majumdar, J. Phys. Condens. Matter 23, 073201 (2011).

[16] Ł. Frackowiak, P. Kuświk, M. Urbaniak, G.D. ChavesO'Flynn, F. Stobiecki, Sci. Rep. 8, 16911 (2018).

[17] M.H. Tang, Z.Z. Zhang, S.Y. Tian, J. Wang, B. Ma, Q.Y. Jin, Sci. Rep. 5, 10863 (2015).

[18] P.D. Kulkarni, A. Thamizhavel, V.C. Rakhecha, A.K. Nigam, P.L. Paulose, S. Ramakrishnan, A.K. Grover, Europhys. Lett. 86, 47003 (2009).

[19] P.D. Kulkarni, U.V. Vaidya, S.K. Dhar, P. Manfrinetti, A.K. Grover, J. Phys. D Appl. Phys. 42, 082001 (2009).

[20] H. Adachi, H. Ino, Nature 401, 148 (1999).

[21] A.K. Nayak, M. Nicklas, S. Chadov, et al., Nat. Mater. 14, 679 (2015).

[22] S. Tsunashima, S. Masui, T. Kobayashi, S. Uchiyama, J. Appl. Phys. 53, 8175 (1982).

[23] C.M. Lee, L.X. Ye, J.M. Lee, W.L. Chen, C.Y. Huang, G. Chern, T.H. Wu, IEEE. Trans. Magn. 45, 3808 (2009).

[24] M. Ding, S.J. Poon, J. Magn. Magn. Mater. 339, 51 (2013)

[25] N. Roschewsky, C.H. Lambert, S. Salahuddin, Phys. Rev. B 96, 064406 (2017).

[26] R. Shan, J. Du, X.X. Zhang, L. Sun, W.W. Lin, H. Sang, T.R. Gao, S.M. Zhou, Appl. Phys. Lett. 87, 102508 (2005)

[27] M. Nakayama, T. Kai, N. Shimomura, M. Amano, E. Kitagawa, T. Nagase, M. Yoshikawa, T. Kishi, S. Ikegawa, H. Yoda, J. Appl. Phys. 103, 07 A710 (2008).
[28] F. Hellman, E.M. Gyorgy, Phys. Rev. Lett. 68, 1391 (1992).

[29] P. Hansen, C. Clausen, G. Much, M. Rosenkranz, K. Witter, J. Appl. Phys. 66, 756 (1989).

[30] C.D. Stanciu, F. Hansteen, A.V. Kimel, A. Kirilyuk, A. Tsukamoto, A. Itoh, T. Rasing, Phys. Rev. Lett. 99, 047601 (2007).

[31] S. Mangin, M. Gottwald, C.H. Lambert, et al., Nat. Mater. 13, 286 (2014).

[32] K. Vahaplar, A.M. Kalashnikova, A.V. Kimel, et al., Phys. Rev. Lett. 103, 117201 (2009).

[33] K. Wang, R. Chen, Y. Huang, J. Non-Cryst. Solids 450, 82 (2016).

[34] N. Bergeard, A. Mougin, M. Izquierdo, E. Fonda, F. Sirotti, Phys. Rev. B 96, 064418 (2017).

[35] T. Hatori, M. Okuda, S. Nakagawa, J. Appl. Phys. 99, 08C513 (2006).

[36] D.J. Webb, A.F. Marshall, Z. Sun, T.H. Geballe, R.M. White, IEEE. Trans. Magn. 24, 588 (1988).

[37] I.V. Golosovsky, M. Estrader, A. López-Ortega, et al., Appl. Mater. Today 16, 322 (2019).

[38] M. Murakami, M. Birukawa, J. Magn. Magn. Mater. 320, 608 (2008).

[39] H. Basumatary, J. Arout Chelvane, D.V. Sridhara Rao, S.V. Kamat, R. Ranjan, Thin Solid Films $\mathbf{5 8 3}$, $1(2015)$

[40] X. Li, C.T. Ma, J. Lu, A. Devaraj, S.R. Spurgeon, R.B. Comes, S.J. Poon, Appl. Phys. Lett. 108, 012401 (2016).

[41] B. Mutelet, N. Keller, S. Roux, et al., Appl. Phys. A 105, 215 (2011). 